\title{
A Feedback-based Molecular Communication Protocol for Noisy Intrabody Environments
}

\author{
Jonathan S. Mitzman*, Bria Morgan*, Torna Omar Soro*†, Junichi Suzuki* and Tadashi Nakano ${ }^{\ddagger}$ \\ *Department of Computer Science \\ University of Massachusetts, Boston \\ Boston, MA 02125, USA \\ Email: $\{$ tolkien,bmorgan,tsoro,jxs\}@cs.umb.edu \\ ${ }^{\dagger}$ History and Social Sciences Department \\ Bunker Hill Community College \\ Boston, MA 02129-2925, USA \\ Email: tosoro@bhcc.mass.edu \\ $\ddagger$ Graduate School of Frontier Biosciences \\ Osaka University \\ Osaka, Japan, 565-0871 \\ Email: tadasi.nakano@fbs.osaka-u.ac.jp
}

\begin{abstract}
This paper considers short-range (up to $100 \mu \mathrm{m}$ ) molecular communication where bio-nanomachines transmit and receive molecule-encoded messages and applies Stop-and-Wait Automatic Repeat Request (SW-ARQ) for feedback-based reliable communication in noisy intrabody environments. Three coommunication transports are considered: (1) diffusive transports where molecules diffuse via random thermal motion, (2) directional transports where molecules directionally move on pre-defined protein filaments with molecular motors and (3) diffusive-directional hybrid transports where molecules propagate with both diffusive and directional transports. Simulation results demonstrate that SW-ARQ improves latency and reliability in both diffusive and directional transports. Hybridization of the two transports aids extra improvements in latency and reliability.
\end{abstract}

\section{INTRODUCTION}

Advances in biological science and nanotechnology make it possible to engineer biologically-enabled functional machines that perform simple computation, sensing and/or actuation tasks on nanometer to micrometer scale by interacting with biological molecules and cells. Those machines, hereafter referred to as bio-nanomachines, may be modified biological cells, artificial cells, which are cell-like artificial structures engineered with biological materials (e.g., a vesicle composed of proteins), or hybrids of artificial nanostructures and biological materials such as DNA strands [1], [2].

An emerging design strategy for nanoscale systems is to network bio-nanomachines for operating in larger physical spaces in higher spatial/temporal resolution. Although individual bio-nanomachines are limited in computation, sensing and actuation capabilities, an assembly of bio-nanomachines can potentially organize into a "large-scale" network that spreads on micrometer to millimeter scale and collaboratively performs tasks that no individual bio-nanomachines could.

Molecular communication is one of a few options to network bio-nanomachines. It leverages molecules as information carriers between bio-nanomachines [3], [4]. Due to its advantages such as inherent nanometer scale, biocompatibility and energy efficiency, molecular communication it is expected to enable various biomedical and healthcare applications such as in-body physiological sensing, biomedical anomaly detection, targeted drug delivery, medical surgeries with cellular/molecular-level precision, artificial morphogenesis and neural signal transduction [4]-[7].

This paper focuses on short-range (up to $100 \mathrm{mum}$ ) molecular communication where bio-nanomachines transmit and receive information molecules (molecules representing information) in aqueous environments. Three communication transports are considered for molecule propagation: (1) diffusive transports where information molecules diffuse through the environment via random thermal motion, (2) directional transports where information molecules directionally move on pre-defined protein filaments (e.g., microtubules) by using molecular motors such as kinesin, dynein and myosin, and (3) diffusive-directional hybrid transports where molecules propagate with both diffusive and directional transports.

Inherent characteristics of molecular communication in aqueous environments are stochastic molecular propagation and environmental noise. They cause environmental congestion by molecules and interference (i.e., collisions) among molecules, which often lead to the losses and out-of-sequence deliveries of information that molecules carry. This requires a communication protocol for reliable molecule propagation.

This paper examines Stop-and-Wait Automatic Repeat Request (SW-ARQ) [8] for reliable short-range molecular propagation for diffusive, directional and diffusive-directional hybrid transports in noisy environments. It exploits a two-way channel: one for transmitter (Tx) bio-nanomachines to propagate information molecules to receiver $(\mathrm{Rx})$ bio-nanomachines and the other one for $\mathrm{Rx}$ bio-nanomachines to propagate acknowledgement (ACK) molecules to Tx bio-nanomachines. Upon receiving an ACK molecule, a Tx bio-nanomachine can recognize that an information molecule, which corresponds to the ACK molecule, has arrived at its destination and initiate the next round of information molecule propagation. 
This paper proposes a particular SW-ARQ protocol for reliable molecule transmissions and evaluates it with biologicallyfeasible parameter settings. Simulation results show that the proposed protocol improves the latency and reliability performance in both diffusive and directional transports. Hybridization of diffusive and directional transports aids extra performance improvements in latency and reliability.

\section{BACKGROUND AND RELATED WORK}

Applications of molecular communication are considered in biomedical, healthcare, environmental and manufacturing domains [5], [6]. This section briefly summarizes how it may be applied to biomedical and healthcare domains.

Lab-on-a-chip: Chemical analysis of biological samples is performed on a chip with dimensions in the millimeter to centimeter range for diagnosis of diseases and other scientific purposes. Molecular communication provides a means to transport specific molecules from sensory components (e.g., biosensors) in a chip to other components in the chip (e.g., chemical reactors). In a potential implementation, a sensory component binds specific molecules in a sample to a certain "interface" molecule or places the molecules in an organelle. A molecular motor carries the interface molecule or organelle and moves on a microtubule from a sensory component to a chemical reactor component [9]. Molecular communication may have advantages because it uses molecular-level mechanisms for directly manipulating the molecules in a sample and does not require translation of information to/from electrical signals. In addition, molecular communication may allow labon-a-chip applications to scale further down since molecular communication components can be at the nanometer scale.

Physiological monitoring: A specific type of molecules can serve as a bio-marker for a disease or a certain physiolosical condition in the body. Implanted bio-nanomachines may exploit biosensors to detect specific molecules and utilize molecular communication as a means of gathering information about the detected molecules, aggregating the information and delivering it to subdermal devices, which in turn communicate to on-body external devices [5].

Artificial morphogenesis: Bio-nanomachines made of living cells can divide and grow to form a three-dimensional multicellular structure such as tissues and organs. Molecular communication allows those bio-nanomachines to communicate with each other using the molecules that encode morphological information as artificial morphogens (AMs). It can affect the growth and differentiation of bio-nanomachines into specific structures by controlling patterns of communication such as the order of AMs to be propagated and the interval of AM propagation. For example, as Tx bio-nanomachines propagate AMs based on a controlled pattern, Rx bio-nanomachines may detect a particular concentration range of the AMs and differentiate into different cell types, resulting in an emerged pattern of differentiated Rx bio-nanomachines. Molecular communication has a potential to advance regenerative medicine.

In the area of molecular communication, major research efforts have been made on the issues in the physical layer, such as channel capacity and physical characteristics of molecular communication (e.g., delay, signal attenuation and energy requirements), using diffusive and directional transports (e.g., [4], [9], [10]). This paper sits on these existing work to investigate a higher-layer issue: feedback-based reliable molecule transmission.

There exist a few relevant work to enhance the reliability of short-range molecular communication in aqueous environments [11]-[14]. Nakano et al. [11] and Felicetti et al. [12] study feedback-based rate control schemes for molecule propagation in diffusive transports. Those schemes are designed to ensure delivering a given number of information molecules to the $\mathrm{Rx}$ while preventing the $\mathrm{Tx}$ from transmitting the molecules faster than the $\mathrm{Rx}$ reacts. While in-sequence delivery of information molecules is out of the scope in [11], [12], this paper focuses on an in-sequence and at-least-once delivery semantics, assuming this semantics is beneficial in such applications as described above. As a result, the proposed protocol explicitly acknowledges information molecules captured by the Rx. (Implicit acknowledgement is used in [11], [12].)

In-sequence molecular transmission is studied with SWARQ schemes for diffusive transport in [13] and bacterial communication in [14]. This paper is similar to [13], [14] in that the two papers utilize SW-ARQ. In addition to diffusive transport, this paper examines directional and diffusive-directional hybrid transports in noisy environments where noise molecules exist and molecules collide with each other. Noisy environments are not considered in $\lceil 11\rceil-\lceil 14\rceil$.

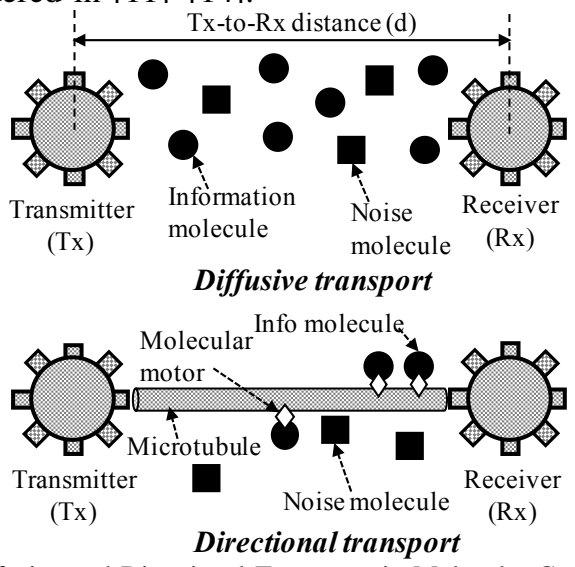

Fig. 1. Diffusive and Directional Transports in Molecular Communication

\section{Molecular Communication Models}

Fig. 1 shows an architectural overview of diffusive and directional transports in short-range molecule communication in noisy aqueous environments. It consists of information molecules that represent the messages (information) to be transmitted, Tx bio-nanomachines that release information molecules, Rx bio-nanomachines that detect and capture information molecules, noise molecules that interfere (i.e., collide) with information molecules, and the environment that molecules propagate through.

Each information molecule encodes a certain message and carries it from the Tx to the Rx. They need to be chemically stable and robust against environmental noise and interference from other molecules. Examples of information molecules include endocrine hormones, local mediators such as cytokines, neurotransmitters (e.g., dopamine and histamine), intracellular messengers (e.g., $\mathrm{Ca}^{2+}$ and cyclic AMP) and DNA/RNA molecules. This paper assumes modified DNA molecules as uniquely identifiable information molecules that can contain specific messages by means of nucleic acid sequences. 
Promising approaches to engineer bio-nanomachines include the modification of biological cells and the production of artificial cell-like structures using biological materials (e.g., a vesicle embedded with proteins) [4]. This paper assumes that bio-nanomachines are realized as modified biological cells, which are known to potentially possess various communication-related functions including a transmission function to synthesize and release specific molecules, a reception function to capture molecules, logic gates to trigger programmed chemical responses upon receiving molecules, toggle switches (i.e., 1-bit memories) to retain communication-related states (e.g., ready-to-transmit and in-transmission/waiting states), and oscillators (i.e., clocks) to control the temporal timing of releasing molecules.

This paper assumes a pure random walk for molecule propagation in diffusive transport. $\mathrm{Rx}$ is assumed to capture an information molecule when it has a physical contact with the molecule. A directional transport requires a microtubule in between $\mathrm{Tx}$ and $\mathrm{Rx}$ to guide information molecules attached to molecular motors. Each microtubule serves as a unidirectional transport. (The microtubule in Fig. 1 is unidirectional from Tx to Rx.) Microtubules are assumed to be stiff, straight and fixed-length cylinders.

This paper considers bounded aqueous environments that contain stationary noise molecules. When an information molecule is diffusing, its movement is governed by the diffusion coefficient $D=\partial x^{2} /(2 \times \partial t)$ independently in three dimensions. $x$ denotes the length of movement and $t$ denotes time. When an information molecule contacts a noise molecule, it randomly moves to another position with $D$. On a microtubule, an information molecule walks at a constant velocity for an expected length (distance walking along a microtubule before randomly diffusing away). It also diffuses away when it collides with a noise molecule on a microtubule. While diffusing, an information molecule with a molecular motor(s) may contact a microtubule and begin to walk along the microtubule. In both diffusive and directional transports, information molecules collide with each other.

\section{SW-ARQ FOR MOLECULAR COMMUNICATION}

SW-ARQ is an error-control method to ensure that all messages are delivered from $\mathrm{Tx}$ to $\mathrm{Rx}$ in the correct order [8]. It is the simplest ARQ method, which is feasible enough to realize with simple computation and memory functions in modified biological cells.

The proposed SW-ARQ protocol allows the Tx to send out $n$ information molecules that encode a particular message. When $n>1$, the Tx propagates duplicated $n$ copies of an information molecule at a time. $n=3$ in an example in Fig. 2. After sending a message, the Tx does not send any further messages until it receives an acknowledgement (ACK) molecule from the Rx. Upon receiving an information molecule, the Rx transmits $n$ ACK molecules, each of which indicates that the $\mathrm{Rx}$ has correctly received a message that the information molecule encodes. In the proposed protocol, the $\mathrm{Rx}$ acknowledges a message once it receives at least one of information molecules that encode the message. The Tx can confirm that a message has arrived at the $\mathrm{Rx}$ when it receives at least one ACK molecules that correspond to the message. In Fig. 2, the Rx acknowledges the arrival of Message 1 since it receives one of three information molecules that encode Message 1. The Tx confirms that Message 1 has arrived at the $\mathrm{Rx}$ by receiving one of three ACK molecules.

Upon receiving an ACK molecule, the Tx transmits $n$ information molecules that encode the next message (e.g., Message 2 in Fig. 2). If it does not receive an ACK molecule that acknowledges the message in a certain timeout interval, it retransmits another set of $n$ information molecules for the message until it receives an ACK molecule or exceeds a predefined number of retransmissions. This interval period is called retransmission timeout interval (RTO). In Fig. 2, Message 2 arrives at the $\mathrm{Rx}$ with a retransmission.

If the $\mathrm{Rx}$ does not receive any information molecules that encode the $i$-th message in RTO after sending ACK molecules for the $(i-1)$-th message, it retransmits another set of $n$ information molecules for the $(i-1)$-th message. In Fig. 2, the Rx retransmits ACK molecules for Message 2 because it receives no information molecules for Message 3 in RTO.

This paper applies SW-ARQ to diffusive, directional and hybrid transports. In a diffusive transport, both information and ACK molecules diffuse in the environment. In a directional transport, two microtubules are deployed to transmit information and ACK molecules. In a directional-diffusive hybrid transport, information and ACK molecules propagate with a directional and diffusive transports, respectively. In each transport, all types of molecules including ACK molecules collide with each other.

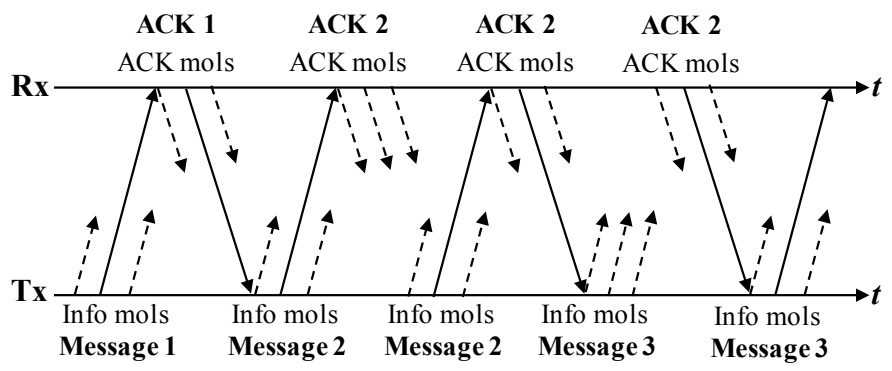

Fig. 2. An Example Sequence of Molecule Transmissions with SW-ARQ. A Solid Line: Successful Molecular Transmission. A Dotted Line: Failed Molecular Transmission.

\section{Simulation Evaluation}

This section evaluates the proposed SW-ARQ protocol in its latency and reliability through simulations. Table I shows a set of simulation parameter settings, which follow findings in biomedical engineering (e.g., [15], [16]). Every result is shown based on 1,000 independent simulations.

TABLE I. PARAMETER Settings

\begin{tabular}{|l|c|}
\hline Parameter & Value \\
\hline \hline Size of the environment & $150 \mu \mathrm{m} \mathrm{x} 150 \mu \mathrm{m} \times 150 \mu \mathrm{m}$ \\
\hline Diameter of Tx and Rx & $5 \mu \mathrm{m}$ \\
\hline Tx to Rx distance & $10,50,70$ and $90 \mu \mathrm{m}$ \\
\hline Diameter of an information molecule & $1 \mu \mathrm{m}$ \\
\hline Diameter of a noise molecule & 0 and $10^{5}$ \\
\hline Number of noise molecules & 0.5 \\
\hline Diffusion coefficient $(D)$ & $1 \mu \mathrm{m} / \mathrm{s}$ \\
\hline Velocity of a molecular motor on a microtubule & $4 \mu \mathrm{m}$ \\
\hline $\begin{array}{l}\text { Expected travel distance of } \\
\text { a molecular motor on a microtubule }\end{array}$ & 5 \\
\hline Max number of molecule retransmissions & 10,20 and 30 \\
\hline \# of molecules propagated per message $(n)$ & \\
\hline
\end{tabular}

Figs. 3 and 4 show how the number of redundant molecules and the existence of noise molecules impact the roundtrip time 
(RTT) between Tx and Rx, which are located 30 and $90 \mu \mathrm{m}$ apart, respectively, in diffusive transports. For example, SWARQ-30 means that Tx sends out 30 redundant information molecules for each message and Rx sends out 30 redundant ACK molecules for each acknowledgement. RTT sums up the time that one of redundant information molecules first hits $\mathrm{Rx}$ and the time that one of redundant ACK molecules first hits Tx. Molecule retransmissions never occur $(\mathrm{RTO}=\infty)$. Fig 5 depicts the median RTT under different Tx-Rx distance settings. Figs. 3 to 5 indicate that RTT increases as noise level and Tx-Rx distance increase. RTT improves as the redundancy in molecule propagation increases. Fig. 5 shows that, using SW-ARQ-30, the median RTT increases from 2,857 to 29,728 seconds as Tx-Rx distance $(d)$ increases from 30 to $90 \mu \mathrm{m}$.

Figs. 6 to 8 illustrate the impacts of molecule redundancy and noise levels on RTT in directional transports. Besides using directional transports, all configurations are same as the ones used for Figs. 3 to 5. RTT increases as noise level and $d$ increase. RTT improves as redundancy increases. With SWARQ-30, the median RTT grows from 186 to 18,978 seconds as $d$ increases from 30 to $90 \mu \mathrm{m}$. In comparison to diffusive transports, directional transports aid to significantly reduce RTT because of directional molecules movement.

Figs. 9 to 11 show how SW-ARQ-30 improves RTT in diffusive transports if molecule retransmission is enabled $(\mathrm{RTO} \neq \infty)$. Four different RTO settings are examined. The first setting is to double the median RTT (RTO=2*RTT). Each of the other settings is configured with the median RTT ("RTT") and the standard deviation of RTT ("STD"). For example, the second RTO setting is to sum up the median RTT and the standard deviation of RTT (RTO=RTT+STD). The maximum number of retransmissions is 5. In comparison to Figs. 3 to 5, Figs. 9 to 11 demonstrate that molecule retransmissions aid to improve RTT. In Fig. 11, the best median RTT is 2,107 and 26,583 seconds when $d=30 \mu \mathrm{m}$ and $d=90 \mu \mathrm{m}$. Molecule retransmissions gain $1.26 \mathrm{x}$ and $1.11 \mathrm{x}$ speedups, respectively.

Figs. 12 to 14 show how hybridization of directional and diffusive transports impacts RTT. Besides this hybridization, all configurations are same as the ones used for Figs. 9 to 11. SW-ARQ-30 yields lower RTT in directional-diffusive hybrid transports than diffusive transports. In Fig. 14, the best median RTT is 910 and 24,202 seconds when $d=30 \mu \mathrm{m}$ and $d=90 \mu \mathrm{m}$. Hybridization gains $1.57 \mathrm{x}$ and $1.09 \mathrm{x}$ speedups respectively.

Table II illustrates the number of messages whose RTTs exceed RTO and 5*RTO in SW-ARQ-30 under four different RTO settings. It verifies that no messages are lost in all transports with all RTO settings if up-to-five retransmissions are enabled. In contrast, without retransmissions, 722 and 464 messages are lost in diffusive and directional transports, respectively, when $\mathrm{RTO}=2 * \mathrm{RTT}$. SW-ARQ improves the reliability of message transmissions.

\section{CONCLUSION}

This paper focuses on short-range molecular communication and applies SW-ARQ for the development of a feedbackbased reliable communication protocol in noisy intrabody environments. Simulation results demonstrate that SW-ARQ improves latency and reliability in both diffusive and direc-
TABLE II. IMPACTS OF RETRANSMISSIONS ON RELIABILITY OF MOLECULE TRANSMISSION

\begin{tabular}{|c|c|r|r|r|r|}
\hline \multirow{2}{*}{} & \multicolumn{4}{|c|}{ RTT $>$ RTO } \\
\cline { 3 - 6 } \multicolumn{2}{|c|}{} & 2*RTT & RTT+ $\frac{1}{3}$ STD & RTT+ $\frac{1}{2}$ STD & RTT+STD \\
\hline \hline \multirow{2}{*}{ Diffusive } & w/ retrans & 457 & 1049 & 843 & 453 \\
\cline { 2 - 6 } & w/o retrans & 722 & 1438 & 1229 & 751 \\
\hline \hline Directional & w/o retrans & 464 & 952 & 801 & 492 \\
\hline \hline Hybrid & w/ retrans & 324 & 904 & 718 & 360 \\
\hline
\end{tabular}

\begin{tabular}{|c|c|r|r|r|r|}
\hline \multirow{2}{*}{} & \multicolumn{4}{|c|}{ RTT $>5 *$ RTO } \\
\cline { 3 - 6 } \multicolumn{2}{|c|}{} & $2 *$ RTT & RTT $+\frac{1}{3}$ STD & RTT $+\frac{1}{2}$ STD & RTT+STD \\
\hline \multirow{2}{*}{ Diffusive } & w/ retrans & 0 & 0 & 0 & 0 \\
\cline { 2 - 6 } & w/o retrans & 50 & 43 & 20 & 3 \\
\hline \hline Directional & w/o retrans & 27 & 30 & 21 & 6 \\
\hline \hline Hybrid & w/ retrans & 0 & 0 & 0 & 0 \\
\hline
\end{tabular}

tional transports. Hybridization of the two transports aids extra improvements in latency and reliability.

\section{REFERENCES}

[1] A. Goel and V. Vogel, "Harnessing biological motors to engineer systems for nanoscale transport and assembly," Nat. Nanotechnol., vol. 3, pp. 465-475, 2008.

[2] P. E. M. Purnick and R. Weiss, "The second wave of synthetic biology from modules to systems," Nat. Rev. Mol. Cell Bio., vol. 10, no. 6, pp. 410-422, 2009.

[3] T. Suda, M. Moore, T. Nakano, R. Egashira, and A. Enomoto, "Exploratory research on molecular communication between nanomachines," in Proc. ACM Genetic and Evol. Computat. Conference, 2005.

[4] T. Nakano, M. Moore, F. Wei, A. V. Vasilakos, and J. W. Shuai, "Molecular communication and networking: opportunities and challenges," IEEE Trans. NanoBiosci., vol. 11, no. 2, pp. 135-148, 2012.

[5] Y. Moritani, S. Hiyama, and T. Suda, "Molecular communication for health care applications," in IEEE Int'l Conf. Pervasive Comput. Commun. Workshops, 2006.

[6] I. Akyildiz, F. Brunetti, and C. Blazquez, "Nanonetworks: a new communication paradigm," Comput. Netw., vol. 52, 2008.

[7] J. Suzuki, D. H. Phan, and H. Budiman, "A nonparametric stochastic optimizer for TDMA-based neuronal signaling," IEEE Trans. NanoBioscience, vol. 13, no. 3, pp. 244-254, 2014.

[8] H. O. Burton and D. D. Sullivan, "Errors and error control," Proc. of the IEE, vol. 60, no. 11, 1972.

[9] N. Farsad, A. Eckford, S. Hiyama, and Y. Moritani, "A simple mathematical model for information rate of active transport molecular communication," in IEEE INFOCOM Wksp Mol Nanosc Comm, 2011.

[10] M. J. Moore and T. Suda, "Molecular communication: Modeling noise effects on information rate," IEEE Trans. NanoBiosci., vol. 8, no. 2, 2009.

[11] T. Nakano, Y. Okaie, and A. V. Vasilakos, "Transmission rate control for molecular communication among biological nanomachines," IEEE J. Sel. Area Comm., vol. 31, no. 12, pp. 835-846, 2013.

[12] L. Felicetti, M. Femminella, G. Reali, T. Nakano, and A. V. Vasilakos, "TCP-like molecular communications," IEEE J. Sel. Area Comm., vol. 32, no. 12, pp. 2354-2367, 2014.

[13] X. Wang, M. D. Higgins, and M. S. Leeson, "Simulating the performance of SW-ARQ schemes within molecular communications," Simulation Modelling Practice and Theory, vol. 42, 2014.

[14] C. Bai, M. S. Leeson, and M. D. Higgins, "Performance of SW-ARQ in bacterial quorum communications," Nano Commun. Netw., vol. 6, no. $1,2015$.

[15] D. E. Smith, T. T. Perkins, and S. Chu, "Dynamical scaling of dna diffusion coefficients," Macromolecules, vol. 29, 1996.

[16] R. D. Vale, T. Funatsu, D. W. Pierce, L. Romberg, Y. Harada, and T. Yanagida, "Direct observation of single kinesin molecules moving along microtubules," Nature, vol. 380, 1996. 


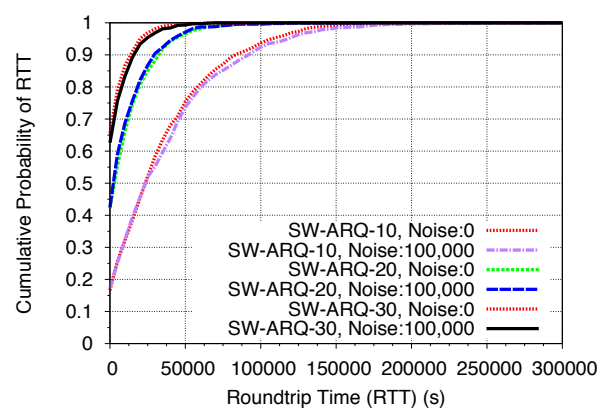

Fig. 3. Cumulative Probability of RTT in Diffusive Transport. $d=30 \mu \mathrm{m}$. RTO $=\infty$.

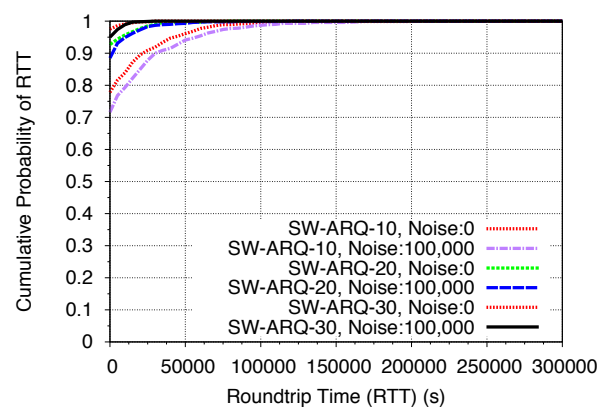

Fig. 6. Cumulative Probability of RTT in Directional Transport. $d=30 \mu \mathrm{m}$. RTO $=\infty$.

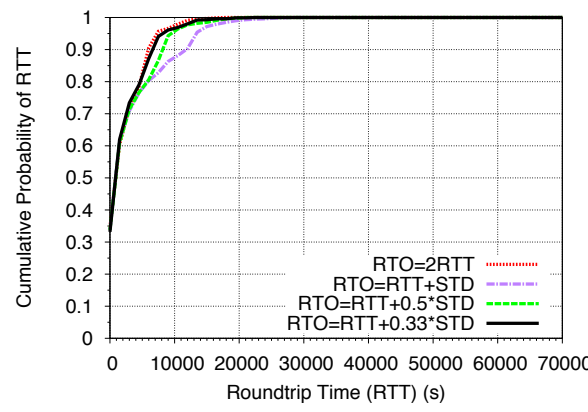

Fig. 9. Cumulative Probability of RTT in Diffusive Transport w/ SW-ARQ-30. $d=30 \mu \mathrm{m}$. Noise: $10^{5}$.

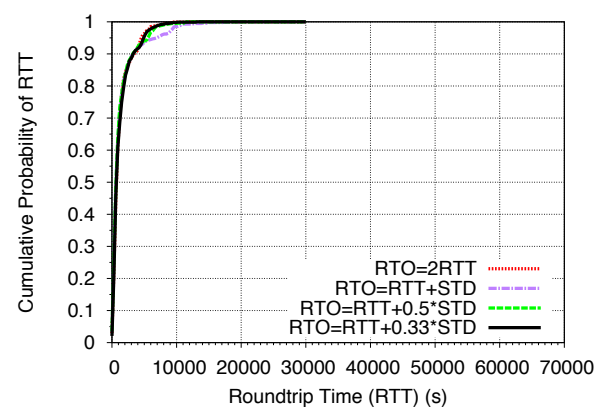

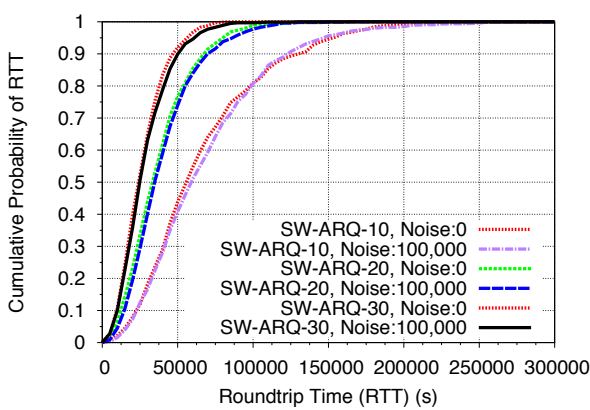

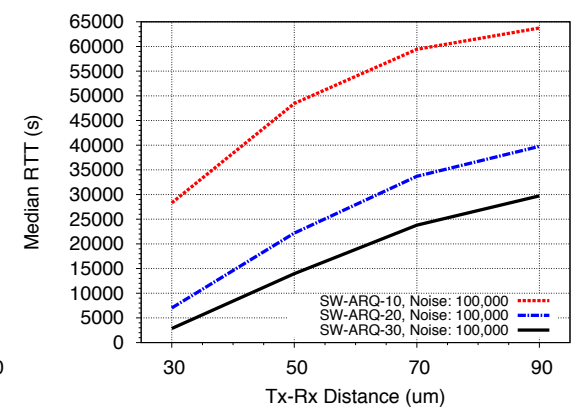

Fig. 4. Cumulative Probability
Transport. $d=90 \mu \mathrm{m}$. RTO= $\infty$.

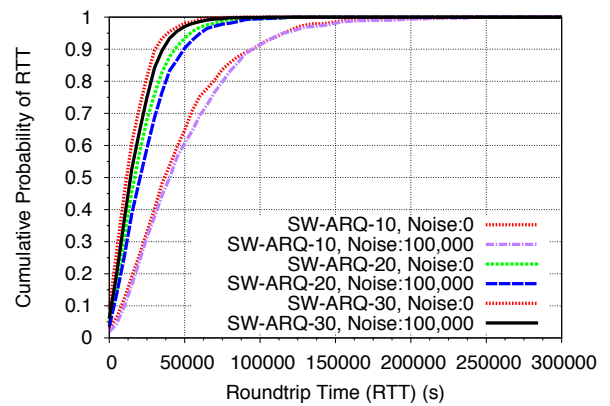

Fig. 7. Cumulative Probability of RTT in Direc- Fig. 8 tional Transport. $d=90 \mu \mathrm{m}$. RTO $=\infty$.

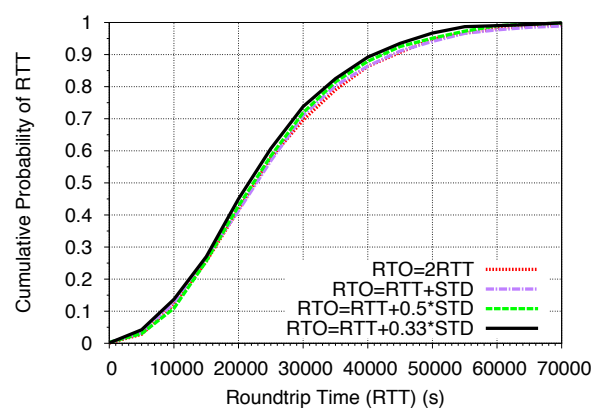

$\mathrm{RTO}=\infty$

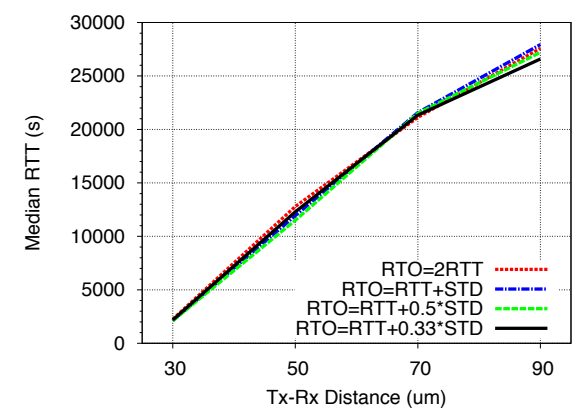

Fig. 5. Median RTT in Diffusive Transport $\mathrm{RTO}=\infty$.

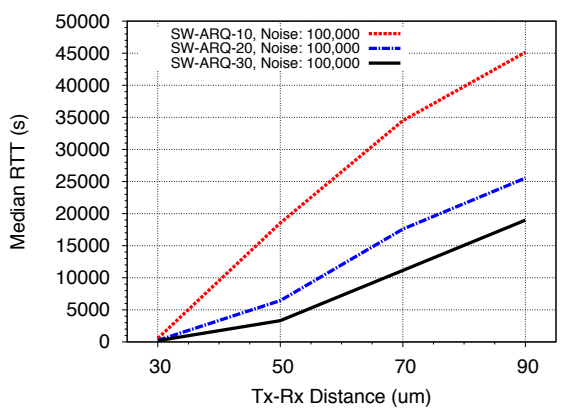

Fig. 10. Cumulative Probability of RTT in Diffusive Fig. 11. Median RTT in Diffusive Transport w/ Transport w/ SW-ARQ-30. $d=90 \mu \mathrm{m}$. Noise: $10^{5}$.

SW-ARQ-30. Noise: $10^{5}$
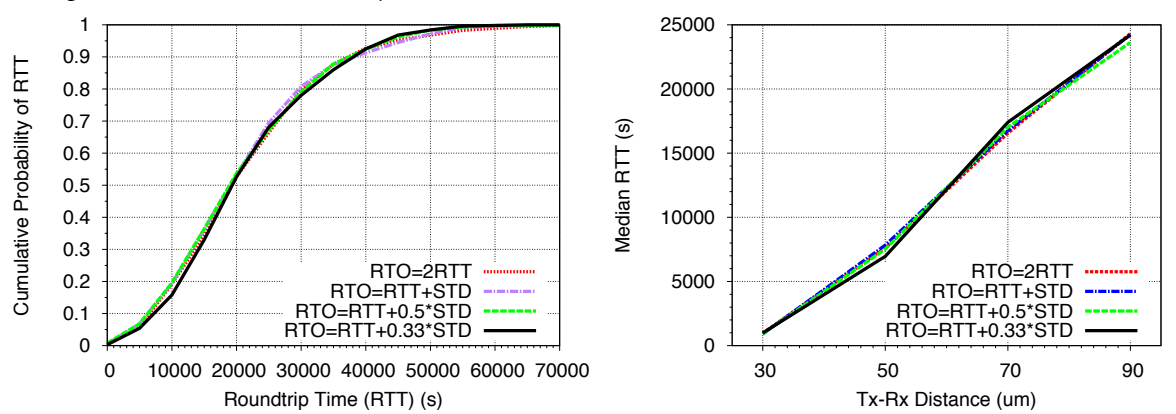

Fig. 12. Cumulative Probability of RTT in Fig. 13. Cumulative Probability of RTT in Fig. 14. Median RTT in Duffusive-Directional Diffusion-Directional Hybrid Transport. SW-ARQ- Diffusion-Directional Hybrid Transport. SW-ARQ- Hybrid Transport. SW-ARQ-30. Noise:10 ${ }^{5}$. 30. $d=30 \mu \mathrm{m}$. Noise: $10^{5}$. 